Baeza Reyes, A. y Lamadrid Álvarez, S. (2018). Trayectorias educativas según género. Lo invisible para la política educativa chilena. Revista de Investigación Educativa, 36(2), 471-490.

DOI: http://dx.doi.org/10.6018/rie.36.2.298061

\title{
Trayectorias educativas según género. Lo invisible para la política educativa chilena
}

\author{
Educational trajectories by gender: \\ The invisible to the Chilean educational policy
}

\author{
Andrea Baeza Reyes y Silvia Lamadrid Álvarez \\ Departamento de Sociología. Facultad de Ciencias Sociales. Universidad de Chile (Chile)
}

\begin{abstract}
Resumen
Chile se encuentra en un momento de reformas de política educativa para contrarrestar su marcada desigualdad, pero la perspectiva de género sigue al margen de la toma de decisiones. Más allá del acceso igualitario a las escuelas, diversos indicadores nacionales e internacionales muestran la persistencia de brechas de aprendizaje entre hombres y mujeres y cómo éstas se traducen en perjuicios para ellas cuando acceden al mercado laboral. En este contexto, realizamos un estudio cualitativo en una escuela de Santiago de Chile, sobre los discursos de estudiantes $y$ docentes de Enseñanza Media acerca de las trayectorias educativas de hombres y mujeres. Gran parte de estos agentes educativos se refieren de manera acrítica a la segregación en las elecciones vocacionales de las y los jóvenes, y el desarrollo de autonomía económica para las mujeres es puesto en duda principalmente por los varones, aun cuando declaran cambios en

Correspondencia: Andrea Baeza Reyes, Socióloga, Universidad de Chile. Miembro del Núcleo de Género Julieta Kirkwood y del Comité Editorial de la Revista Punto Género, Universidad de Chile. Sus líneas de investigación son sociología del género, medios de comunicación y educación. correo andreaa.baeza@gmail. com, Universidad de Chile, Facultad de Ciencias Sociales. Departamento de Sociología, Ignacio Carrera Pinto 1045, Ñuñoa. Santiago. Región Metropolitana, Chile.

Silvia Lamadrid Álvarez, Socióloga, Pontificia Universidad Católica de Chile. Doctora en Historia, mención Historia de Chile, Universidad de Chile. Académica del Departamento de Sociología de la Universidad de Chile, donde coordina el Núcleo de Género Julieta Kirkwood. Editora de la Revista Punto Género. Sus líneas de investigación son sociología del género, acción colectiva, historia del género, correo: silamadr@u. uchile.cl, Universidad de Chile, Facultad de Ciencias Sociales. Departamento de Sociología, Of. 316. Ignacio Carrera Pinto 1045, Ñuñoa. Santiago. Región Metropolitana, Chile.
\end{abstract}


los roles de género movilizados precisamente por la educación. Se constata además, una escasez de estrategias formales al interior de la escuela para abordar la temática, en contraste con el interés del cuerpo docente por formar de manera más integral a sus estudiantes. Ello permite debatir la eficacia de la actual política educativa en Chile y proponer acciones de mejora para generar trayectorias en igualdad.

Palabras clave: género; educación; transición enseñanza secundaria-enseñanza superior; política de la educación.

\section{Abstract}

Chile is in a moment of educational policy reforms to counteract its strong inequality, but the gender perspective remains marginalized to the decision making. Beyond equal access to schools, many national and international indicators show the persistence of learning gaps between men and women and how these differences become detriment for women when they entry to job market. In this context, we did a qualitative study in a school of Santiago de Chile with secondary's students and teachers on educational trajectories of men and women and their consequences on the job market. Most educational agents' discourses refer to segregation in vocational elections of youth in an uncritical way. Besides, men are the ones that mainly criticize the economic autonomy for women even when it indicates changes in gender roles promoted by education. Also, it is confirmed a lack of formal strategies inside the school to approach this issue, which contrasts with the teachers' interest in giving an integral training to their students. This allows for the debate about the effectiveness in the current educational policy in Chile and to propose some improvement actions to generate trajectories in equality.

Keywords: gender; education; transition secondary education-higher education; educational policy.

\section{Introducción}

En Chile existen avances significativos en materia de cobertura educativa. Desde el siglo pasado, niñas y mujeres han tenido un acceso gradual a todos los niveles de enseñanza escolar, pasando de ser una población excluida a constituir hoy la mitad de la matrícula en el sistema escolar (Ministerio de Educación de Chile [Mineduc] 2013; Ministerio de Desarrollo Social, 2012). Considerando estos avances, aún permanecen importantes representaciones estereotípicas al interior de las aulas, obstáculos para el desarrollo con igualdad de género.

Desde los años setenta, la escuela ha sido un escenario clave para los estudios con perspectivas de género (Salomone, 2007). Es señero el estudio de Acker (1995), en que, desde su experiencia en Estados Unidos e Inglaterra, presenta las teorías feministas sobre género y educación y las perspectivas de una Sociología de la Educación de la Mujer. Analiza los contextos familiares y profesionales de las mujeres dedicadas a la educación, desde el nivel básico hasta el superior, abordando críticamente las discriminaciones, exclusiones y marginaciones que encuentran. Demuestra, fundadamente, que en las sociedades estudiadas el género influye desfavorablemente en la educación de las mujeres. 
El reciente estudio de las investigaciones rigurosas sobre educación y género considera el análisis de intervenciones en variados países, ya sea en los recursos e infraestructura, en el cambio institucional o en el cambio de normas para la inclusión. Respecto a este último ámbito menormente documentado, las mujeres que acceden a la educación superior mejoran las nociones sobre sí mismas y su participación en el espacio público, y sutilmente definen sus propias aspiraciones y toman el control de sus elecciones de vida. Los autores concluyen que "una mayor disposición de oportunidades educativas en sí mismas no llevan a un mayor acceso al mercado laboral" (Unterhalter et al., 2014, p. 70), y que es necesario que las políticas tengan un compromiso permanente con la educación para promover el empoderamiento en las niñas y las jóvenes.

Otras investigaciones internacionales han analizado las diferencias de género dentro de las prácticas y discursos de una determinada asignatura (Devis, Fuentes, \& Sparkes, 2005; Scharagrodsky, 2004). En España, Cerezo y Casanova (2004) examinan las diferencias de género existentes en distintas variables cognitivo-motivacionales y en el rendimiento alcanzado en las asignaturas de Lenguaje y Matemáticas; las estudiantes presentan menores niveles de motivación extrínseca, se responsabilizan más de sus fracasos, utilizan en mayor medida estrategias de procesamiento de la información y obtienen mejores calificaciones en Lenguaje. Costa y Tabernero (2012) identifican diferencias de género en el rendimiento académico y en el autoconcepto en estudiantes de Educación Secundaria Obligatoria de España, a favor de las mujeres en las asignaturas de Lengua Española y Literatura, en tanto los estudiantes varones poseen un autoconcepto positivo no sólo en general sino también en las facetas académica, social, emocional, familiar y física, lo cual influye en su rendimiento académico. García-Pérez et al. (2010) se enfocaron en el estudio de las actitudes del alumnado hacia la igualdad, encontrando actitudes del alumnado favorables hacia la igualdad, aunque se observan diferencias entre chicos y chicas.

Delgado, Inglés, García-Fernández, Castejón y Valle (2010) analizaron las diferencias en metas académicas de estudiantes de Educación Secundaria Obligatoria, encontrando que estas variaron significativamente según el género y el curso de los estudiantes, siendo entre las mujeres la orientación motivacional mayor hacia metas de aprendizaje y metas de logro, mientras los jóvenes obtuvieron un mayor patrón motivacional hacia metas de refuerzo social. Colás y Jiménez-Cortés (2006) se enfocaron en los tipos de conciencia de género que tiene el profesorado en los centros escolares de secundaria, encontrando tipos de conciencia de género diferenciados en función del sexo de los profesores, así como, variables asociadas a los mismos.

En Chile, algunas investigaciones se han centrado en desentrañar el sexismo en los textos escolares chilenos (Covacevich \& Quintela-Dávila, 2014; Montecino, 1997; Palestro, 2016), sin embargo "no existe una producción considerable de estudios relativos a las diferencias de género al interior de las escuelas" (Madero, 2011, p. 137).

Estudios de la realidad escolar en el contexto nacional (Flores, 2005; Parra, 1997; Valdés, 2013) e internacional (Devis et al., 2005; Mosteiro \& Porto, 2017; Scharagrodsky, 2004; Stromquist, 2006) tensionan la eficacia de los esfuerzos para la eliminación de discriminación entre hombres y mujeres dentro de estas instituciones en relación a los avances societales. Estos coinciden en las discrepancias entre el currículo formal, basado en los ideales democráticos de la meritocracia, y el currículo oculto de la docencia y 
las dinámicas escolares sesgadas, que desde muy temprano establecen diferencias jerarquizantes entre "lo masculino" y "lo femenino". A saber, "el lenguaje y las prácticas docentes siguen estando imbuidas de un estilo tradicional, (...) que reafirma la estructura, roles y funciones tradicionales atribuidas a hombres y mujeres, en el marco de relaciones asimétricas" (Caro, González, \& Zegpi, 2001, pp. 177-178).

La escuela perfila y legitima ciertos ideales y deseos (...), participa en la formación de las "promesas de felicidad" de la época, con lo cual va conformando una trama de representaciones que persuaden a los/as estudiantes a desear ocupar determinados lugares sociales, y a aceptar un orden social y de género que se presenta como natural, verdadero y racional (Flores, 2005, p. 74).

Puesto que toda la evidencia internacional indica que la escuela es un sistema que pone en diálogo a los distintos agentes más allá de las materias pedagógicas y está en estrecha relación con los valores de la sociedad donde se inserta, es relevante conocer las representaciones sociales de género que allí se configuran, en un sentido amplio, interconectado las diversas asignaturas y hablantes.

Esto adquiere relevancia no solo con el propósito de disminuir las diferencias de aprendizaje en las distintas pruebas nacionales e internacionales, las que muestran cómo las disparidades tienden a ampliarse a medida que se avanza en el sistema escolar (UNESCO, 2015), sino, sobre todo, porque estando en la misma aula, leyendo los mismos libros y escuchando al mismo profesor o profesora, niños y niñas, o bien, las y los jóvenes reciben educaciones muy diferentes y ello se traduce en trayectorias educativas disímiles, afectando la autonomía y al desarrollo de las mujeres (Sadker, como se citó en Chapman, 2005).

Conforme a lo anterior, el presente trabajo indaga en los saberes y experiencias de estudiantes y docentes acerca de las trayectorias educativas que realizan y podrían realizar hombres y mujeres jóvenes en una escuela secundaria mixta de Santiago de Chile.

\section{Mujeres y educación, más allá de la cobertura del sistema educativo}

En el siglo pasado, inicialmente la educación constituía un bien escaso y reservado a los sectores económicos altos y a los varones lo que se modificó paulatinamente por los avances de la educación (Veneros, 2001).

La legislación sobre los mínimos exigibles en educación para la población chilena (Ley de Instrucción Primaria Obligatoria creada en 1920, modificada en 1929, 1965, 2003 y 2015) en la actualidad fijan en trece los años de enseñanza obligatorios, incluyendo un año en pre-escolar, ocho en primaria y cuatro en secundaria (Treviño, Villalobos, \& Baeza, 2016). Con ello, se han aumentado las tasas de cobertura. En educación básica ésta alcanza el 98\% y en educación media el 95\%, sin una variación significativa por sexo (Mineduc, 2017). La tasa de graduados de la Educación Media en Chile es equivalente al promedio OCDE (83\%) y dentro de esta cifra, son más mujeres (86\%) que hombres $(80 \%)$ quienes terminan el ciclo escolar completo (Mineduc, 2013).

Conforme los cambios en las políticas educativas han ampliado las oportunidades y el acceso al sistema escolar, es indudable que "las mujeres han ido ganando mayor 
acceso a la educación en comparación con el pasado" (Stromquist, 2006, p. 370). El problema radica en que estas políticas enmarcan la igualdad de oportunidades en el acceso a los establecimientos educativos.

Tener un número semejante de mujeres y varones en las aulas no asegura que ambos grupos tengan las mismas oportunidades al interior de estas. Se viene constatando que existen diferencias de rendimiento académico por género en diversas pruebas internacionales (TIMSS, PISA) y nacionales (SIMCE), donde los hombres logran mejores rendimientos en matemáticas y ciencias y las mujeres en lectura (Agencia de la Calidad de la Educación, 2011, 2012, 2013).

\section{Puntajes máximos PSU según género}

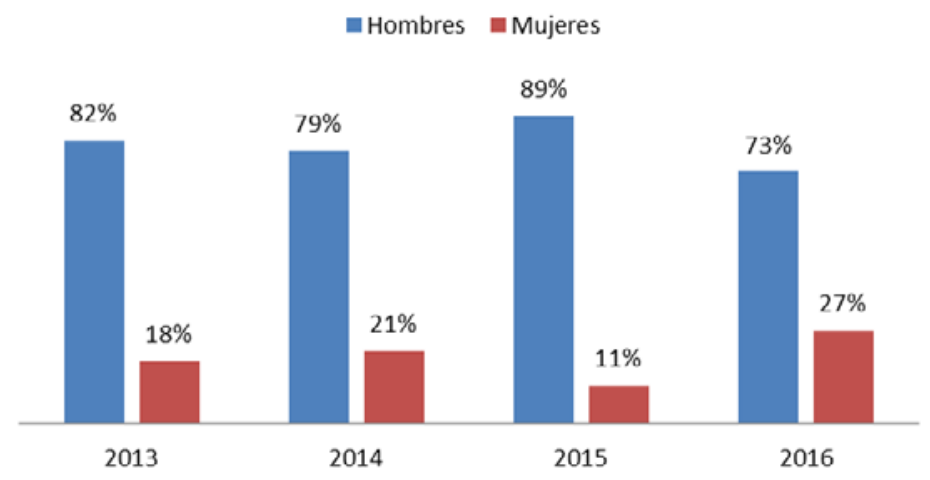

Figura 1. Puntajes Máximos en la Prueba de Selección Universitaria según género.

Una situación particular se observa respecto a la Prueba de Selección Universitaria (PSU), examen necesario para acceder a gran parte de las instituciones de educación superior en Chile. Existen diferencias en cuanto a la obtención de puntajes máximos (850 puntos) según género en los últimos años (ver Figura 1). Estos son obtenidos mayoritariamente por varones a nivel nacional, lo que tiene gran incidencia en el acceso a la educación superior, puesto que las personas con mejores puntajes -además de las calificaciones de Enseñanza Media- tienen mayores posibilidades de elegir una carrera acorde a sus intereses, además de acceder a los beneficios socioeconómicos del Estado y de dichas instituciones

La paridad en el acceso a las escuelas tampoco asegura, una vez terminado el ciclo escolar, que las y los jóvenes realicen las mismas trayectorias educativas. Esto es, la continuación de estudios de nivel superior, dadas las disposiciones culturales favorables y las promesas de movilidad social asociadas (Sepúlveda \& Valdebenito, 2014).

La "elección académico-profesional es resultado de un conjunto de variables, esfuerzos, aspiraciones e intereses personales” (Ricoy \& Sánchez-Martínez, 2016, p. 301). Por encima de los factores asociados al estudiantado -el desempeño académico, vocación expectativas familiares- está la gestión escolar y sus acciones de apoyo para asegurar trayectorias exitosas. Se debe considerar de manera integral las acciones de tipo formal e informal que realizan la escuela y sus agentes, para acompañar a los y las estudian- 
tes en sus transiciones educativas (Williamston, 2010). En un mayor nivel, están las políticas educativas que condicionan a ambos, puesto que la estructura institucional puede afectar los procesos transicionales dentro del sistema escolar, de manera directa o indirecta (Treviño et al. 2016).

Recientes estudios muestran cómo, una vez terminado el ciclo escolar, las trayectorias de las y los jóvenes siguen una clara diferenciación por motivos de género, afectando su desarrollo en equidad (González-Badilla, 2016). En particular, el rol que continúan ejerciendo las mujeres como madres, afecta su motivación, intereses y aspiraciones (Ricoy \& Sánchez-Martínez, 2016). Si bien las niñas y jóvenes se desempeñan bien en la escuela, incluso a veces mejor que sus compañeros, "terminan siendo en su gran mayoría dueñas de casa a tiempo completo o bien trabajadoras pobres" (Rossetti, como se citó en Parra, 1997, p. 38), ocupando lugares secundarios en la estructura productiva tanto en términos de remuneraciones como de responsabilidades.

En Chile se constata una alta segregación según áreas y carreras en la educación superior (Papadópulos \& Radakovich, 2006; Parra 1997; Valdés, 2013). Las carreras altamente demandadas por mujeres son enfermería, obstetricia, fonoaudiología, trabajo social y educación, servicios de cuidado considerados típicamente femeninos, con baja remuneración económica. Los hombres ingresan de manera predominante en carreras vinculadas a las ciencias físicas y matemáticas. Es baja la proporción de mujeres matriculadas en primer año en el área de tecnología (21.6\%), y preeminente en el área de educación (71.5\%) y ciencias sociales (68.9\%) (Consejo Nacional de Educación [CNED], 2015).

Esta desigualdad arrastrada desde la escuela, además tiene consecuencias en el mercado de trabajo. Precisamente, "las diferencias entre hombres y mujeres en la elección de las carreras es uno de los factores que conducen a un mercado laboral segregado por género" (Mizala, 2014, p.11).

La equidad de género forma parte de los Objetivos del Milenio y de los Derechos Humanos. Desde los lineamientos internacionales se plantea explícitamente que "las consideraciones de género deben instalarse en todas las políticas y programas, incluyendo ámbitos políticos, económicos y sociales, para que favorezcan de manera equitativa a hombres y mujeres" (ECOSOC, como se citó en UNESCO, 2014, p. 101).

La Convención sobre la Eliminación de Todas las Formas de Discriminación contra la Mujer (en inglés CEDAW) ratificada por Chile en 1989, estipula la importancia de asegurar la igualdad de derechos y oportunidades en la educación para hombres y mujeres (art.10 en ONU, 1979). En 2000 se realizó el Foro Mundial de Educación Dakar, cuyo marco de acción inscribió las particulares desventajas de las mujeres en comparación a los hombres en el sistema educacional. En esta materia se recalcó que el desafío para la equidad trasciende la escolarización, ya que esta no es sino una igualdad virtual (UNESCO, 2014).

So pretexto del potencial transformador de la escuela con la exposición de ideas cosmopolitas y consideraciones éticas para los individuos, es menester volver a mirar la institución escolar considerando los valores y pautas de comportamientos que mantienen y refuerzan representaciones sociales de género que pudieran estar afectando el futuro educacional, profesional y de inserción laboral de las jóvenes, así como también el desarrollo de su autonomía y empoderamiento. 


\section{Método}

\section{Objetivo}

Nuestro objetivo es conocer e indagar en los saberes y experiencias de docentes y estudiantes acerca de las trayectorias educativas que realizan y podrían realizar hombres y mujeres jóvenes en una escuela secundaria mixta de Santiago de Chile.

\section{Contexto y casos}

Siguiendo el criterio de tipicidad (Valles, 1999), el contexto seleccionado fue una escuela particular subvencionada, mixta, de enseñanza científico-humanista y de estrato medio bajo. En estricto rigor, se buscó una escuela "típica" de Chile que no se financiara exclusivamente por el aporte del Estado, cuyo proyecto educativo no fuese alternativo respecto a los estándares el Mineduc, que no recibiera al reducido número estudiantes de sectores acomodados y considerando la situación socioeconómica del establecimiento, tampoco se tratase de una escuela emblemática o de excelencia académica. A este contexto se tuvo acceso en la comuna de La Cisterna de Santiago.

En la escuela la centralidad fue el ciclo Enseñanza Media (EM), por la importancia que tiene para la configuración de la identidad juvenil y porque posee cambios curriculares sustantivos en comparación a la anterior enseñanza básica, para una posible continuación de estudios de nivel superior. La EM será considerada un microsistema de (re)producción de sentidos, valores y opiniones acerca de los géneros, el cual es susceptible de conocer de manera inferencial a partir del habla experiencial y social cotidiana de los agentes en interacción (Canales, 2006).

Los casos dentro de esa institución responden a una selección intencionada mediante consideraciones teóricas que permitan abrir la heterogeneidad desde distintas perspectivas discursivas dentro del contexto (Valles, 1999). A partir del criterio de relevancia intrínseca (Mejía, 2004) el muestreo teórico consideró a docentes hombres y mujeres que impartieran variadas disciplinas (humanistas, científicas y recreativas) y a estudiantes de ambos géneros que pertenecieran a los cuatro cursos $\left(1^{\circ}\right.$ a $\left.4^{\circ}\right)$ de la Enseñanza Media. Además, se estableció como requisito que la permanencia de docentes como estudiantes fuera de por lo menos un semestre, tiempo considerado mínimo para adquirir las experiencias necesarias de arraigo y pertenencia, como también de internalización de normativas, reglas de convivencia social y académica, y el acercamiento a los valores que promueven los actores del establecimiento. Los hablantes que participaron de la investigación fueron 5 docentes, 9 estudiantes. Sus características se detallan a continuación en Tabla 1 y Tabla 2.

Se construyó un guión temático con cinco dimensiones (Tabla 3) que fue empleado en cinco entrevistas individuales semi-estructuradas con las y los docentes, un focus group con las estudiantes -Focus de Mujeres (FM)- y otro focus group con los estudiantes -Focus de Hombres (FH)-.

Los temas abordados fueron currículo oculto, currículo y propuestas de abordaje, los que permitieron indagar en los saberes y prácticas (formales e informales) propios de los agentes de la escuela. Considerando que la desventaja sistemática por razones de género existe más allá de esta esfera (Smith, 2014), también se incluyeron preguntas 
acerca de los roles de género al interior de las familias y experiencias o percepciones sobre el mercado de trabajo. Este guión fue revisado por expertas metodológicas antes de su aplicación en su versión final.

Tabla 1

Muestra definitiva de docentes

\begin{tabular}{|c|c|c|}
\hline Criterios & Hombre & Mujer \\
\hline Disciplina Humanista & $\begin{array}{l}\text { Profesor de Historia y Geografía, } 34 \\
\text { años y en la escuela hace } 1 \text { año y medio }\end{array}$ & \\
\hline Disciplina Científica & $\begin{array}{l}\text { Profesor de matemáticas, } 47 \text { años, y en } \\
\text { la escuela hace } 4 \text { años }\end{array}$ & $\begin{array}{l}\text { Profesora de Biología, } 39 \\
\text { años, y en la escuela hace } \\
13 \text { años }\end{array}$ \\
\hline Disciplina Deportiva & $\begin{array}{l}\text { Profesor de Educación Física, } 48 \text { años y } \\
\text { en la escuela hace } 18 \text { años }\end{array}$ & $\begin{array}{l}\text { Profesora de Educación } \\
\text { Física, } 28 \text { años y en la es- } \\
\text { cuela hace } 8 \text { meses }\end{array}$ \\
\hline
\end{tabular}

Tabla 2

Muestra definitiva de estudiantes

\begin{tabular}{lcc}
\hline \multicolumn{1}{c}{ Criterios } & Alumnas & Alumnos \\
\hline $1^{\circ} \mathrm{EM}$ & Una estudiante (14 años) & Un estudiante (15 años) \\
$2^{\circ} \mathrm{EM}$ & Dos estudiantes (ambas de 16 años) & Dos estudiantes (16 y 17 años) \\
$3^{\circ} \mathrm{EM}$ & Una estudiante (17 años) & Dos estudiantes (17 y 18 años) \\
\hline
\end{tabular}

Instrumento

Tabla 3

Guión temático

\begin{tabular}{ll}
\hline Dimensión & \multicolumn{2}{l}{ Indicadores } \\
\hline Familia & 1. ¿Cuáles son los roles de género en las familias? \\
& 2. ¿Qué sucede con los roles de hombres y mujeres hoy en día, en com- \\
& paración a décadas atrás? (Indagar si se han mantenido o han cambiado \\
& o ambas)
\end{tabular}


Currículo oculto

Currículo y propuestas de abordaje

6. ¿Existen carreras más adecuadas para mujeres y otras para hombres? ¿Cuáles? ¿Por qué?

7. ¿Cómo se tratan o abordan estos temas en la escuela? Si no, ¿cómo podrían hacerlo?

8. (Para docentes) ¿Cuál cree que es su papel en la formación de género al interior de su escuela?

9. (Para docentes) ¿Ud. cree que en su asignatura se abordan estos temas? (¿De qué forma? ¿En qué se suele enfatizar?)

10. ¿Cuán importante es discutir sobre género en la escuela?

\section{Procedimiento de recogida y análisis de datos}

La investigación se realizó previa aprobación del equipo directivo del establecimiento. Luego se les ofreció a los y las participantes los respectivos consentimientos y asentimientos. En estos documentos se les solicitó aprobar la grabación en audio de la sesión de conversación para su posterior transcripción y análisis. Junto a ello, se estableció el uso de las opiniones estrictamente para propósitos de este estudio.

El levantamiento de información se realizó entre junio y julio de 2014. Este estudio fue descriptivo, de tipo no experimental (Hernández, Fernández, \& Sampieri, 2010).

Sobre el material transcrito, realizamos un análisis de contenido, el que por medio de procedimientos sistemáticos, permite el análisis e interpretación de los sentidos latentes y manifiestos expresados en un texto, en referencia a su contexto de producción (Navarro \& Díaz, 1999).

\section{Resultados}

A continuación presentamos los resultados obtenidos aludiendo, en primera instancia, a las trayectorias educativas; y enseguida, cómo éstas se relacionan con las concepciones en torno al rol proveedor. Luego, cuál es la educación (¿o el sesgo?) de género que las y los hablantes reconocen dentro de la escuela y finalmente, sus propuestas para abordarla (ver Figura 2).

\section{Trayectorias educativas}

Todas las personas entrevistadas reconocen diferencias de género en la elección de carreras profesionales. Las y los estudiantes sostienen que "sí hay una diferencia de estudios porque las mujeres no se enfocan mucho tampoco a la construcción, se enfocan a otras cosas, y los hombres tampoco se enfocan a las cosas que se enfocan las mujeres (...) los hombres apuntan a (ser) ingenieros" (FH). La realidad observada y la experiencia de cercanos les indican que "la mayoría de las parvularias hoy día son mujeres" (FM), o bien que en carreras como ingenierías "hay súper pocas mujeres" (FH). 
En la mujer es típico (estudiar para) parvularia, o profesora, secretaria, peluquera, pero el hombre es mecánico, o tiene algún local no sé de insumos de computación o de cosas así, pero a un hombre es raro verlo en parvularios y a una mujer es raro verla en construcción (FM).

El profesor de historia señala que hay "carreras más adecuadas para mujeres" como la educación diferencial, asistentes de la educación, o secretariado, bibliotecología, donde "se ve mucho la llegada de mujeres" (E1).

El docente de matemáticas señala sobre sus alumnas, que "básicamente van al área humanista o biología”, "también por Lingüística o quieren estudiar Derecho. De cuarto medio (...) de las 9 que hay a ninguna le he escuchado que quiera estudiar matemáticas" (E3). Sus estudiantes varones, en cambio, se inclinan por matemáticas o Ingeniería Comercial. En general, "el hombre va por Arquitectura, a pesar que igual hay mujeres arquitectas" (E3).

En educación física, la profesora y el profesor comparten las ideas mencionadas. A saber, "enfermería es una carrera donde tú siempre ves a la enfermera, hay enfermeros, sí, pero yo creo que ahí el porcentaje es minoritario" (E5) y en construcción hay más varones. En esta misma línea, "ver a una mujer estudiando ingeniería civil es como raro" (E4). "A las mujeres casi siempre les gusta la parte más humanista, no tanto científico" (E4).

La profesora de biología enfatiza que la elección de carreras por las mujeres es más diversa. A ellas "les gusta la medicina a otras les gusta la psicología, que a otras les gusta el trabajo social, como que son más amplias en ese sentido, en cambio los hombres son como más cuadrados, en la parte ingeniería, arquitectura, como de ahí, son pocos los que salen de esa área" (E2).

El profesor de historia sostiene que "la mujer tiene más (...) capacidades y mayores facilidades para tratar con niños más chicos y si no se ha notado la entrada de hombres (en educación de párvulos) es por algo" (E1).

Desde la asignatura deportiva, ésta tendencia estriba en las capacidades y manera de tomar decisiones asociadas: "ellos tienen más capacidades, son más racionales, pueden tomar decisiones más rápido, las mujeres no, a las mujeres les cuesta tomar decisiones, son más indecisas, los hombres no, son más directos, toman decisiones rápido" (E4).

Para la docente de biología la razón de las elecciones vocacionales corresponde a las habilidades, argumentando sobre sus ex alumnos que entraron a la carrera de Ingeniería, que "tenían más aptitudes, les gustaban las matemáticas, la física" (E2).

Las explicaciones que ofrecen las y los docentes sobre la segregación por motivos de género en la elección vocacional se sustenta en una concepción de habilidades innatas. Esta mirada sobre las competencias e intereses de las y los estudiantes termina naturalizando las opciones diferenciales de hombres y mujeres y estableciendo a priori caminos paralelos en cuanto a la elección vocacional, donde el de los hombres es eminentemente distinto y opuesto al de las mujeres.

\section{¿Proveedor...as?}

Un elemento emergente de los discursos tanto de estudiantes como docentes en esta escuela, atañe a la familia actual y a la división sexual del trabajo, donde coexisten diversas miradas. 
Las jóvenes señalan que existe cierta apertura respecto a los rígidos roles de género al interior de la familia. "Está muy visto que es el hombre el que tiene que llevar como la plata a la familia, el que tiene que trabajar, y la mujer (es) la que se tiene que quedar en la casa, pero igual ahora esto está cambiando, se están cambiando los roles" (FM).

La profesora de educación física respalda la observación de este cambio social, donde actualmente pareciera que las mujeres pudieran elegir cualquier carrera para continuar sus estudios terciarios. Esto es importante porque "antes la mujer no estudiaba, antes la mujer se quedaba en la casa, hoy en día la mujer estudia y sale de la casa, creo que en ese sentido sí se ha hecho un refuerzo súper positivo en el sentido de que sí podemos estudiar, y podemos trabajar" (E4), superando la dependencia económica.

La profesora de biología relativiza que en las familias actuales el ímpetu de las mujeres trabajadoras no necesariamente pasa por una vocación personal, sino por necesidad de aportar ingresos. Así, "hoy en día llegas y no está la mamá (en la casa como antaño), la mamá está trabajando (remuneradamente...) hay una mayor inserción laboral de la mujer por opción y algunas obligadas" (E2).

Los jóvenes varones, en cambio, ven con mayor crítica el avance de las mujeres hacia el mundo laboral, ya que éste no las libera por sí solo de las responsabilidades domésticas y de cuidado que históricamente han llevado a cabo. Si bien "por generaciones va cambiando la realidad de que las mujeres se vuelven más independientes, pero el estereotipo sigue siendo el mismo, de que la mujer tiene que quedarse en casa y el hombre afuera" (FH).

El profesor de matemáticas comparte la idea que "la carga más pesada se la lleva la mujer. Por ejemplo, si los dos trabajan, la mujer es la que tiene que llegar a cocinar, a limpiar...el papá puede que se preocupe un poco de los hijos" (E3). Conforme a ello, y aunque los hombres están más participativos, duda que exista una co-responsabilidad igualitaria y más aún en las tareas del hogar.

Adicionalmente, también se mencionaron algunas de las dificultades presentes en el mercado laboral para las mujeres. La profesora de educación física señala que los altos cargos directivos son principalmente ocupados por hombres, aunque las mujeres también compitan por obtener dichos cargos. Su colega masculino se refirió a la diferencia de sueldo entre hombres y mujeres en Chile. El profesor de matemáticas mencionó la dificultad de las mujeres para encontrar trabajo en ámbitos masculinos, como en ingenierías.

Los discursos de los/as hablantes se posicionan dentro de una paradigma tradicional en torno a los roles de género en la familia, donde la dicotomía establecida entre el varón proveedor y la mujer encargada del hogar y del cuidado de otras personas queda explicitada, aun cuando reconocen el ingreso -por deseo o necesidad- al mercado de trabajo. Las mujeres en esta última situación presentan doble carga laboral, una remunerada, la otra no.

\section{¿Educación de Género en la escuela?}

El estudiantado señala que no hay alguna acción formal en el sentido de la equidad de género en la educación, sino solamente en sexualidad, con una mirada biológica 
enfocada en la prevención del embarazo adolescente. Las jóvenes indican que es "siempre lo mismo cada año, las drogas, sexualidad, drogas, sexualidad" (FM). El profesor de matemáticas coincide en que el género no se aborda de manera formal, sino que como programa de educación sexual.

Algunos docentes comentan que realizan un trabajo más específico con algunos de los cursos, según sean los contenidos del currículo a impartir. Por ejemplo la profesora de biología trata género e identidad pero lamentando que "no hay mucho tiempo" (E2).

(...) los abordo más que nada como en séptimo y en segundo medio cuando tomo sexualidad y hablamos de la identidad sexual y ahí como que hablamos del género, (...) qué es lo que influye, por qué yo me siento hombre, por qué me siento mujer, qué hago como hombre, qué hago como mujer, y ahí lo desarrollamos un poco más pero tampoco es tan profundo, porque la cantidad de contenidos que uno tiene que pasar es enorme (E2).

En historia y también desde el currículo, el profesor hace referencia explícita a los derechos de las mujeres. "Antes no se enseñaba, quizás antes, treinta o cuarenta años, no se hablaba de los derechos de las mujeres, ahora sí se habla, entonces eso es un avance" (E1).

(...) los movimientos sociales, y muchos de los movimientos sociales son de mujeres, y los cambios institucionales y políticos, también se pasan en cuarto medio y se habla ahí en cuarto medio de la creación de nuevos ministerios, como el ministerio de la mujer. Entonces sí se está enseñando a los alumnos cuales han sido, éste nuevo rol que tiene la mujer, y eso ayuda para que en el futuro, se tenga mayor conciencia (E1) (de los aportes a la sociedad en sus distintos momentos).

Extracurricularmente, la profesora de educación física relata que no ha presenciado alguna actividad formal sobre género y menos acerca de las trayectorias educativas, que incluya a otros actores, como las familias y apoderados.

La carencia de estrategias sistemáticas e institucionalizadas en el currículo o fuera de éste para abordar y promover la igualdad de género es un indicio de que ésta no tiene un carácter prioritario en la escuela. Instalar la sensibilidad hacia las temáticas de género, encaminada hacia el empoderamiento es una tarea compleja y requiere prestar atención a la reforma general del sistema, "al cambio institucional a nivel de escuela y del salón de clases (Unterhalter et al., 2014, p. 75).

\section{Propuestas y desafíos}

Ante la preeminencia de metodologías expositivas poco estimulantes en cuanto a sexualidad, las jóvenes recalcan que preferirían "participar, (en vez de) mirar una powerpoint de lo mismo" (FM) y aprovechar la convivencia escolar para dialogar los temas de género. 
Tematizando su rol de "enseñar y educar" (E3), las y los docentes recalcan que es preciso abordar incluso aquello que todavía no está en los programas. Similarmente, proponen cultivar el respeto y la no discriminación de géneros, como base de una buena convivencia escolar hoy para las próximas generaciones.

También aportan otros elementos importantes a las propuestas de una educación de género en la escuela, orientada a las trayectorias educativas.

El cuerpo docente señala que hay que ser un "guía frente a estas situaciones, frente a estos temas" (E2) que afectan tanto a hombres como mujeres en el presente o que pudieran afectarles en el futuro. Sin embargo, más allá de la voluntad de cada uno, "hay una cosa de tiempo que a nosotros nos juega en contra" (E2). Además, recalcan que depende muchas veces del interés y recursos que inviertan las autoridades de la escuela.

Eso va a depender de los sostenedores de los colegios, en que inviertan en ciertos temas. Yo puedo tener muchas ganas de trabajar (estos temas), pero tiene que venir alguien aquí y hacerles una charla y mostrarle cosas...hay algunos que lo hacen gratis, algunos que cobran. También va a depender de eso (E2).

En esa misma línea, sobre el rol docente es que "nosotros le moldeamos el pensamiento a los niños, también aquí a las familias y la sociedad (...) creamos lo que pasa a futuro" (E4). Por ello, habría que incorporar también a las madres, padres y apoderados en cualquier iniciativa en género que se desee implementar en la escuela. Precisamente, "les podría ayudar el asunto, en hacerles entender de que sus hijos y sus hijas son capaces de hacer lo mismo que hace el compañero del sexo opuesto" (E4).

Es preciso deconstruir miradas hegemónicas sobre los roles de género en la familia y la sociedad chilena, así como también rescatar los aspectos afectivos y de autoestima en el desarrollo de las y los jóvenes que pudiesen afectar sus elecciones vocacionales.

Por último, y a nivel de políticas públicas, sugieren innovar en la formación de profesores, incorporando perspectivas de género en las carreras de pedagogía en Chile.

(El género) debe ser mayormente tratado, y especialmente en las pedagogías, las pedagogías siguen todavía, las mallas curriculares antiguas, eso es lo peor, que en algunas carreras de pedagogía en universidades privadas y tradicionales, todavía no se han actualizado muy bien, (...) para poder enseñar bien esos temas, entonces tiene que haber un cambio estructural en la pedagogía (E1).

En efecto, un cambio institucional efectivo pasa por generar una "mezcla de calidad", esto es "la integración de la igualdad de género en la enseñanza, el aprendizaje y la gestión” (Unterhalter et al., 2014, p. 76). Para ello es preciso atender el programa de estudio, los materiales de aprendizaje y por supuesto, las prácticas pedagógicas. En este marco, es menester incluir la perspectiva de género en los distintos niveles del sistema educativo. 


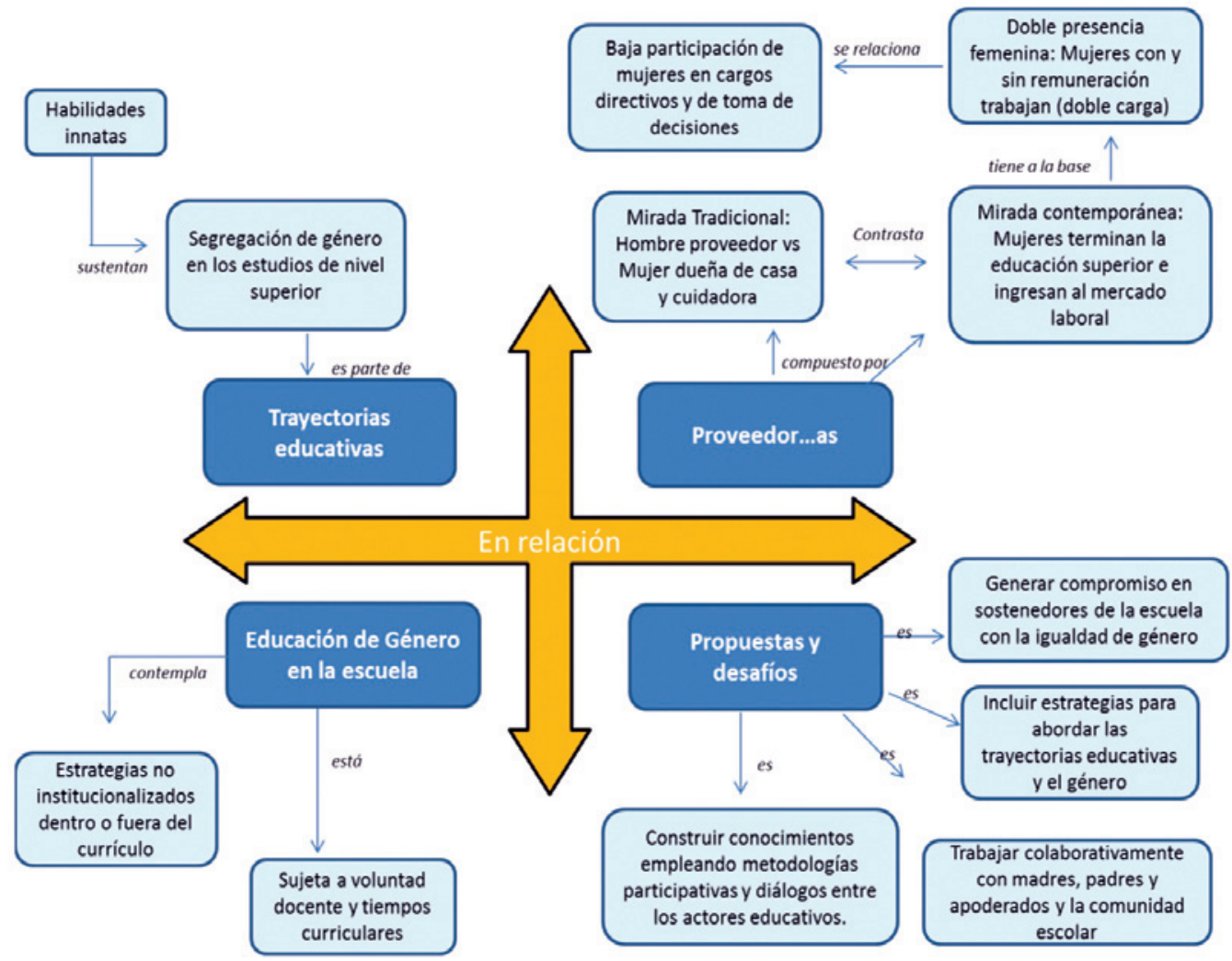

Figura 2. Síntesis categorías y subcategorías (nodos) de los resultados.

\section{Discusión y conclusiones}

A partir de esta investigación, los resultados muestran que en el tránsito de la Enseñanza Media hacia la Educación Superior, profesores y estudiantes de ambos géneros identifican que existen carreras para mujeres (educación de párvulos, diferencial, general básica, secretariado, enfermería) y otras para hombres (construcción, ingenierías, arquitectura, informática). Las explicaciones que sustentan esta distinción son los intereses o habilidades disciplinares entre ellos y ellas, con lo que naturalizan una construcción social sobre las opciones vocacionales indudablemente diferenciadas.

Las mujeres terminan la escuela en mayor proporción que los varones (Mineduc, 2013), y aunque tienen un inferior desempeño en la Prueba de Selección Universitaria, esto no se traduce en un menor acceso a las instituciones de educación superior, pero sí limita su acceso a los establecimientos con mayor prestigio. Considerando las diferencias de calidad, con fuerza desde 2008, se constata la mayor matrícula femenina en universidades que no forman parte del Consejo de Rectores (CRUCH), así como en las instituciones Técnico Profesionales. Aquí las carreras que más demandan son precisamente 
secretariado, educación de párvulos, enfermería y servicio social (Mineduc, 2017), alineadas con los discursos de los agentes escolares a los que se tuvo acceso en este estudio.

Confirmando lo señalado por otros estudios en esta materia (CNED, 2015; Papadópulos \& Radakovich, 2006; Parra, 1997), las elecciones vocacionales para las y los jóvenes siguen una clara diferenciación de género y responden más a razones del aprendizaje sociocultural de éste que a un simple azar (Mizala, 2014). Esta situación, además, está muy poco problematizada, por lo que se perpetúa y se reproduce de manera acrítica dentro de la escuela.

De la sensibilización respecto a la igualdad de género, encontramos que ésta es medianamente compartida por estudiantes y docentes. García-Pérez et al. (2010) sostienen que las alumnas están más sensibilizadas en esta materia, pero en esta investigación, en algunos aspectos los alumnos incluso son más críticos, por ejemplo, sobre la articulación del trabajo remunerado y no remunerado, que no alcanza todavía para la deconstrucción de estereotipos.

Se constata que las expectativas de desarrollo laboral se ven atravesadas con la división sexual del trabajo, donde conviven concepciones progresistas y tradicionales acerca de los roles femeninos y masculinos. El último Estudio Nacional sobre el Uso de Tiempo (2015) revela diferencias en el trabajo doméstico no remunerado para el propio hogar, donde las mujeres destinan el doble de horas en un día normal que los hombres, independiente de su situación laboral (Instituto Nacional de Estadística [INE], 2016). Esta implica una pobreza de tiempo, cuando la doble presencia femenina, no trastoca los roles históricamente asignados de hombre-proveedor/mujer-cuidadora (Baeza, Baeza, \& Ibaceta, 2014). Junto a ello, el porcentaje de mujeres en Chile que participa en el mercado laboral $(48.3 \%)$ es bajo en comparación al promedio de la OCDE (54.1\%) y más aún en relación a los hombres (72\%) (INE, 2015).

Este estudio de caso en una escuela típica (subvencionada-mixta-de estrato social medio-bajo y de enseñanza científico-humanista) de Santiago muestra la escasez de estrategias formales para abordar las trayectorias educativas desde un enfoque de género. Aun cuando existe un alto interés de los y las docentes del establecimiento, este se ve obstaculizado por la falta de tiempo, y dinero de la administración para apoyarles con expertos en esta materia. Futuros estudios cualitativos de caso en contextos urbanos podrían dar cuenta de las estrategias informales que realizan las y los docentes, como son la orientación, motivación y otros apoyos psicosociales dirigidos al estudiantado con miras a las trayectorias educativas acordes a sus expectativas (Williamston, 2010) y observar si se siguen reproduciendo sesgos o estereotipos de género en estas prácticas cotidianas.

La institución escolar estaría afectando la igualdad de oportunidades en la reproducción de estereotipos y expectativas de desarrollo diferenciadas por parte del profesorado hacia los y las alumnas. "Las implicancias de estas trayectorias sesgadas por género es que las mujeres tienden a estar sub-representadas en las profesiones mejor pagadas en el mercado laboral" (Mizala, 2014, p. 10). Esto tiene como consecuencias un mercado laboral segregado por razones de género y la permanencia de las brechas salariales en Chile -oscilante entre 33\% y $26.5 \%$ en favor de los varones (INE, 2015)- socavando las posibilidades reales de autodeterminación y autonomía económica para las mujeres. 
Chile desde 1990 "asumió un compromiso con la superación de las desigualdades entre los sexos, de modo de garantizar a mujeres y hombres un acceso equitativo a los beneficios, recursos y oportunidades de desarrollo" (Valdés, 2013, p. 18).

Los marcos políticos y legales chilenos han intentado colaborar en la construcción de relaciones no discriminatorias entre los géneros. Desde la Política Pública, están las reformas curriculares y pedagógicas (Contenidos Mínimos Obligatorios, Objetivos Fundamentales Transversales, el Marco de la Buena Enseñanza) orientadas a generar cambios culturales sobre las prácticas que producen y reproducen discriminaciones hacia las niñas y mujeres. Además está la paulatina incorporación de las colaboraciones de las mujeres en lo político, económico y cultural en los textos escolares, en la ciencia y en la historia. También se ha incluido el género dentro de la Evaluación docente (Mineduc, 2017).

Pese a estas iniciativas, dentro de las escuelas no existe un amplio reconocimiento de la subordinación de las mujeres, un abordaje de sus causas, ni tampoco la incorporación de "los aportes de las teorías de género en las diferentes disciplinas" (Valdés, 2013, p. 54).

La falta de consensos entre diversos sectores políticos-ideológicos y las relaciones de poder que han tensionado los marcos institucionales del país cuando se han intentado innovaciones, han obstaculizado una política de educación con perspectiva de género (Programa de las Naciones Unidas para el Desarrollo [PNUD], 2010). Hasta la fecha, no se ha implementado una política con este enfoque en los sistemas educativos que aborde este nudo crítico, y cualquier avance pro equidad sigue dependiendo de la interpretación y voluntad de cada establecimiento y de sus docentes.

En Chile aún "existe un vacío en términos de una política de género que atienda las evidentes necesidades educativas de niños, niñas y jóvenes" (Madrid, 2006, p. 18). La Reforma en Educación mantiene el foco en el acceso igualitario a los centros educativos para los sectores vulnerables como garantía de la calidad. La Ley de Inclusión Escolar (Ley 20.845) no especifica el género como dimensión, sino que busca la erradicación de cualquier discriminación arbitraria que ocurra en la escuela. Por ello, es menester complementar las iniciativas mencionadas con otras propuestas que intencionen el cambio hacia la igualdad, como lo están demandando hoy los movimientos feministas de las universidades chilenas.

Las y los profesores en ejercicio como las futuras docentes, requieren de conocimientos y herramientas para desarrollar pedagogías de género de manera sistemática, no aislada ni sujeta a voluntad. Ello implica intervenir la formación inicial y continua, velando por que los contenidos teóricos tengan correlatos empíricos en las prácticas profesionales. Para ello, una medida propuesta por Madrid (2006) ha de ser promover el trabajo de las mujeres en la Educación Media y a los varones en la Educación Básica y Párvulos para empezar a deconstruir la segregación en el ejercicio docente.

En los medios de comunicación, destacamos campañas de sensibilización sobre las trayectorias educativas y opciones vocacionales provenientes de la organización civil Comunidad Mujer durante segundo gobierno de Michelle Bachelet. "Eduquemos con Igualdad" fue lanzada en marzo de 2016 y "Las Niñas Pueden", desde octubre de ese año, la que además cuenta con el apoyo del BID, ONU-Mujeres y la Unión Europea.

Finalmente, para el desarrollo de la igualdad de género el sistema escolar debe construir caminos sin prejuicios ni prácticas discriminatorias. Hay que remirar los sesgos 
y estereotipos del currículo oculto que están arraigados en las prácticas y discursos escolares, implicando a la comunidad escolar de manera integral y otras instituciones, en la participación activa en el diálogo, abordaje e intervención para construir trayectorias educativas sin desigualdades de género.

\section{Referencias}

Acker, S. (1995). Género y educación: reflexiones sociológicas sobre mujeres, enseñanza y feminismo. Madrid: Narcea.

Agencia de la Calidad de la Educación (2011). Resultados TIMSS 2011 Chile. Estudio Internacional de Tendencias en Matemática y Ciencias. TIMSS. Recuperado de http:// portales.mineduc.cl/usuarios/acalidad/doc/201301151653440.Informe_Resultados_ TIMSS_2011_Chile_(10-01-13).pdf

Agencia de la Calidad de la Educación (2012). Informe Nacional Resultados Chile PISA 2012. Recuperado de https://s3.amazonaws.com/archivos.agenciaeducacion.cl/ documentos-web/Estudios+Internacionales/PISA/Informe_Nacional_Resultados_ Chile_PISA_2012.pdf

Agencia de la Calidad de la Educación (2013). Informe Nacional de Resultados. Ampliando la mirada dela calidad educativa. 2013. Recuperado de http://archivos.agenciaeducacion. cl/documentos-web/Informe_Nacional_Resultados_Simce2013.pdf

Baeza, A., Baeza, P., \& Ibaceta, V. (2014). La(s) pobreza(s) desde el género. Una aproximación a la realidad chilena actual. Revista Punto Género, 4(4), 83-105. doi: http://dx.doi.org/10.5354/0719-0417.2014.36410

Canales, M. (2006). Metodología de investigación social. Introducción a los oficios. Santiago de Chile (Chile): LOM.

Caro, A., González, J., \& Zegpi, M. (2001). Mujer, poder y conocimiento. La educación como instrumento de promoción de la igualdad de oportunidades: Una reflexión inicial. En D. Escobar, R. Flores, \& D. Veneros (Eds.). Investigaciones. Red Nacional Universitaria Interdisciplinaria de Estudios de Género (pp. 174-187). Santiago de Chile (Chile): SERNAM.

Cerezo, M., \& Casanova, P. F. (2004). Diferencias de género en la motivación académica de los alumnos de Educación Secundaria Obligatoria. Electronic Journal of Research in Educational Psychology, 2(3), 97-112. Recuperado de http://www.investigacionpsicopedagogica.org/revista/articulos/3/espannol/Art_3_31.pdf

Chapman, A. (2005). Gender Bias in Education. Educational Research, 46(2). Recuperado de http://www.edchange.org/multicultural/papers/genderbias.html

Consejo Nacional de Educación [CNED] (2015). PMG. Estadísticas de Género. Matrícula Primer Año 2015. Índices matricula. Recuperado de http://www.cned.cl/public/ secciones/SeccionIndicesPostulantes/OtrasEstadisticas/Estadisticas_Genero_ Matricula_Primer_2015.pdf

Colás, P., \& Jiménez-Cortés, R. (2006). Tipos de conciencia de género del profesorado en los contextos escolares. Revista de Educación, 340, 415-444.

Costa, S., \& Tabernero, C. (2012). Rendimiento académico y autoconcepto en estudiantes de educación secundaria obligatoria según el género. Revista Iberoamericana de Psicología y salud, 3(2), 175-193. 
Covacevich, C., \& Quintela-Dávila, G. (2014). Desigualdad de género, el currículo oculto en textos escolares chilenos. Banco Interamericano de Desarrollo División de Educación (SCL/ EDU). Recuperado de https://publications.iadb.org/bitstream/handle/11319/6647/ Desigualdad\%20de\%20g\%C3\%A9nero,\%20el\%20curr\%C3\%ADculo\%20oculto\%20 en $\% 20$ textos $\% 20$ escolares $\% 20$ chilenos.pdf?sequence $=1$

Delgado, B., Inglés, C. J., García-Fernández, M., Castejón, J. L., \& Valle, A. (2010). Diferencias de género y curso en metas académicas en alumnos de Educación Secundaria Obligatoria. Revista Española de Pedagogía, 68(245), 67-83.

Devis, J., Fuentes, J., \& Sparkes, A. (2005). ¿Qué permanece oculto del currículum oculto? Las identidades de género y de sexualidad en la educación física. Revista Iberoamericana de Educación, 39, 73-90. Recuperado de https://dialnet.unirioja.es/ejemplar/135704

Navarro, P., \& Díaz, C. (1999). Análisis de Contenido. En J. M. Delgado, \& J. Gutiérrez (Eds.), Métodos y Técnicas cualitativas de investigación en Ciencias Sociales (pp. 177-224). Madrid: Editorial Síntesis.

Flores, R. (2005). Violencia de género en la escuela: sus efectos en la identidad, autoestima y en el proyecto de vida. Revista Iberoamericana de Educación, 38, 67-86. Recuperado de https://dialnet.unirioja.es/ejemplar/128755

García-Pérez, R., Rebollo-Catalán, A., Buzón-García, O., González-Piñal, R., Barragán, R., \& Ruiz-Pinto, E. (2010). Actitudes del alumnado hacia la igualdad de género. Revista de Investigación Educativa, 28(1), 217-232. Recuperado de http://revistas.um.es/ rie/article/view/98951/109441

González-Badilla, P. (2016). Efectos de una educación sexista en la vida de las mujeres. En Red Chilena contra la Violencia hacia las Mujeres (Ed.), No quiero ser princesa, quiero ser científica. Educación no sexista para una real transformación (pp. 25-35). Santiago de Chile (Chile): Red Chilena contra la Violencia hacia las Mujeres.

Hernández, R., Fernández, C., \& Sampieri, P. (2010). Metodología de la Investigación. México: Mc Graw Hill.

Instituto Nacional de Estadística [INE] (2015). Mujeres en Chile y Mercado del trabajo. Participación laboral femenina y brechas salariales. Santiago de Chile (Chile): Instituto Nacional de Estadística. Recuperado de http://nuevoportal.ine.cl/docs/defaultsource/laborales/ene/publicaciones/mujeres-en-chile-y-mercado-del-trabajo--participaci\%C3\%B3n-laboral-femenina-y-brechas-salariales.pdf?sfvrsn=4

INE (2016). ENUT. Encuesta Nacional sobre el Uso de Tiempo. Documento de Principales Resultados ENUT 2015. Santiago de Chile (Chile): Instituto Nacional de Estadística. Recuperado de http://historico.ine.cl/enut/files/principales_resultados/documento_ resultados_ENUT.pdf

Madero, I. (2011). Inclusión y exclusión de género y clase al interior de la escuela chilena en 4 comunas del sur de Chile. Estudios Pedagógicos, 37(2), 135-145. doi: http://dx.doi. org/10.4067/S0718-07052011000200008

Madrid, S. (2006). Profesorado, política educativa y género en Chile: Balance y propuestas. Colección Ideas. Año 8, n76. Recuperado de http://www.clam.org.br/bibliotecadigital/ uploads/publicacoes/257_1700_profesoradopolitica.pdf

Mejía, J. (2004). Sobre la investigación cualitativa. Nuevos conceptos y campos de desarrollo. Investigaciones Sociales, 8(13), 279-299. Recuperado de http:// revistasinvestigacion.unmsm.edu.pe/index.php/sociales/article/view/6928 
Ministerio de Educación de Chile [Mineduc] (2013). Chile en el panorama educacional internacional OCDE: avances y desafíos. Centro de Estudios Mineduc. Serie Evidencias, Año 2, n¹8. Recuperado de http://centroestudios.mineduc.cl/tp_enlaces/portales/ tp5996f8b7cm96/uploadImg/File/Evidencias/A2N18_Chile_en_OECD.pdf

Mineduc (2017). Educación para la Igualdad de Género. Plan 2015-2018. Recuperado de http://www.mineduc.cl/wp-content/uploads/sites/19/2017/01/CartillaUEG.pdf

Ministerio de Desarrollo Social (2012). Educación. Sección Educación Escolar. Recuperado de http://www.ministeriodesarrollosocial.gob.cl/ipos-2012/media/ipos_2012_pp_3065.pdf

Mizala, A. (2014). Mujer y trabajo. Estereotipos y brechas de género en los rendimientos académicos. Serie Comunidad Mujer $n^{\circ} 28$. Recuperado de http://dev.comunidadmujer. cl/biblioteca-publicaciones/wp-content/uploads/2014/06/281.pdf

Montecino, S. (1997). Constructores del afuera y moradoras del interior. Representaciones de lo masculino y de lo femenino en libros escolares chilenos. Una mirada desde la antropología del género a los textos de castellano de enseñanza básica. En B. Eyzaguirre, \& L. Fontaine (Eds.). El futuro en riesgo. Nuestros textos escolares (pp. 503-524). Santiago de Chile (Chile): Centro de Estudios Públicos.

Mosteiro, M.J., \& Porto A.M. (2017). Análisis de los estereotipos de género en alumnado en formación profesional: diferencias según sexo, edad y grado. Revista de Investigación Educativa, 35(1), 151-165. doi: http://dx.doi.org/10.6018/rie.35.1.257191

Palestro, S. (2016). Androcentrismo en los textos escolares. En Red Chilena contra la Violencia hacia las Mujeres (Ed.), No quiero ser princesa, quiero ser científica. Educación no sexista. Hacia una real transformación (pp. 15-23). Santiago de Chile (Chile): Red Chilena contra la Violencia hacia las Mujeres.

Parra, M. E. (1997). La desigualdad según el género y su relación con los ámbitos público y privado. Cinta moebio, 1, 61-76. Recuperado de http://www.cintademoebio.uchile. cl/index.php/CDM/article/viewFile/26486/27780

Programa de las Naciones Unidas para el Desarrollo [PNUD] (2010). Género: los desafíos para la igualdad. Programa de las Naciones Unidas para el Desarrollo Humano en Chile. Santiago de Chile (Chile): PNUD.

ONU (1979) Convención sobre la Eliminación de Todas las Formas de Discriminación contra la Mujer. Adoptada el 8 de diciembre de 1979. Recuperado de http://www.un.org/ womenwatch/daw/cedaw/text/sconvention.htm

Papadópulos, J., \& Radakovich, R. (2006). Educación Superior y Género en América Latina y el Caribe. En IESALC (Eds.), Informe Sobre la Educación Superior en América Latina y el Caribe. La metamorfosis de educación superior (pp. 117-128). Caracas: IESALC.

Ricoy, M. C., \& Sánchez-Martínez, C. (2016). Preferencias académicas y laborales en la adolescencia: Una perspectiva de género. Estudios Pedagógicos, 42(2), 299-313.doi: 10.4067/S0718-07052016000200017

Salas, A. (23 de diciembre de 2016). PSU: E1 73\% de los puntajes nacionales son hombres y sólo el 27\% mujeres. El Mercurio On Line. Recuperado de http://www.emol.com/ noticias/Nacional/2016/12/23/836970/PSU-El-73-de-los-puntajes-nacionales-sonhombres-y-solo-el-27-mujeres.html 
Salomone, R. (2007). Igualdad y diferencia. La cuestión de la equidad de género en la educación. Revista Española de Pedagogía, 238, 433-446. Recuperado de https:// revistadepedagogia.org/wp-content/uploads/2008/05/238-02.pdf

Scharagrodsky, P. A. (2004). Juntos pero no revueltos: la educación física mixta en clave de género. Cadernos de Pesquisa, 34(121), 59-76. doi: http://dx.doi.org/10.1590/ S0100-15742004000100004

Sepúlveda, L., \& Valdebenito, M.J. (2014). ¿Las cosas claras? Aspiraciones de futuro y proyecto educativo laboral de jóvenes estudiantes secundarios. Estudios Pedagógicos, 40(1), 243-261. doi: http://dx.doi.org/10.4067/S0718-07052014000100015

Smith, S. (2014). Limitations to equality: Gender stereotypes and social changes. Juncture, 21(2), 144-150. doi: http://dx.doi.org/10.1111/j.2050-5876.2014.00795.x

Stromquist, N. (2006). Una cartografía social del género en educación. Educação $\mathcal{E}$ Sociedade, 27(95), 361-383. doi: http://dx.doi.org/10.1590/S0101-73302006000200003

Treviño, E., Villalobos, C., \& Baeza, A. (2016). Recomendaciones de Políticas Educativas en América Latina en base al TERCE. Santiago de Chile (Chile): OREALC/UNESCO. Recuperado de http://unesdoc.unesco.org/images/0024/002449/244976s.pdf

UNESCO (2014). América Latina y el Caribe. Reporte Regional 2015 de Revisión de la Educación para Todos. Santiago de Chile (Chile): OREALC/UNESCO. Recuperado de http://unesdoc.unesco.org/images/0023/002327/232701s.pdf

UNESCO (2015). Tercer Estudio Regional Comparativo y Explicativo (TERCE). Factores Asociados. Santiago de Chile (Chile): OREALC/UNESCO. Recuperado de http:// unesdoc.unesco.org/images/0024/002435/243533s.pdf

Unterhalter, E., North, A., Arnot, M., Loyd, C., Moletsane, L., Murphy-Grahan, E., Parkes, J., \& Saito, M. (2014). La educación de las niñas y las jóvenes y la igualdad de género. Análisis de investigaciones rigurosas sobre educación. Londres (UK): Departamento de Desarrollo Internacional del Gobierno del Reino Unido.

Valdés, T. (2013). Género en la escuela, o la porfiada desigualdad. Revista Docencia, 49, 46-61. Recuperado de http://www.revistadocencia.cl/new/wp-content/ pdf/20130626215807.pdf

Valles, M. (1999). Técnicas Cualitativas de Investigación Social. Reflexiones metodológicas y práctica profesional. Madrid: Síntesis.

Veneros, D. (2001). Siglo XX, Cambalache. Breve reseña histórica de los avances de las mujeres en Chile. En D. Escobar, R. Flores, \& D. Veneros (Eds.). Investigaciones. Red Nacional Universitaria Interdisciplinaria de Estudios de Género (pp. 103-121). Santiago de Chile (Chile): SERNAM.

Williamston, R. (2010). Transition from middle school to high school. Pittsburg, Pensilvania (EE.UU): Education Partnerships, Inc. Recuperado de http://files.eric.ed.gov/fulltext/ ED538706.pdf

Fecha de recepción: 28 de junio de 2017

Fecha de revisión: 30 de junio de 2017

Fecha de aceptación: 13 de febrero de 2018 